Document downloaded from:

http://hdl.handle.net/10251/121345

This paper must be cited as:

Ossorio-García, J.; Vague Cardona, JJ.; Boria Esbert, VE.; Guglielmi, M. (2018). Exploring the Tuning Range of Channel Filters for Satellite Applications Using Electromagnetic-Based Computer Aided Design Tools. IEEE Transactions on Microwave Theory and Techniques. 66(2):717-725. https://doi.org/10.1109/TMTT.2017.2769083

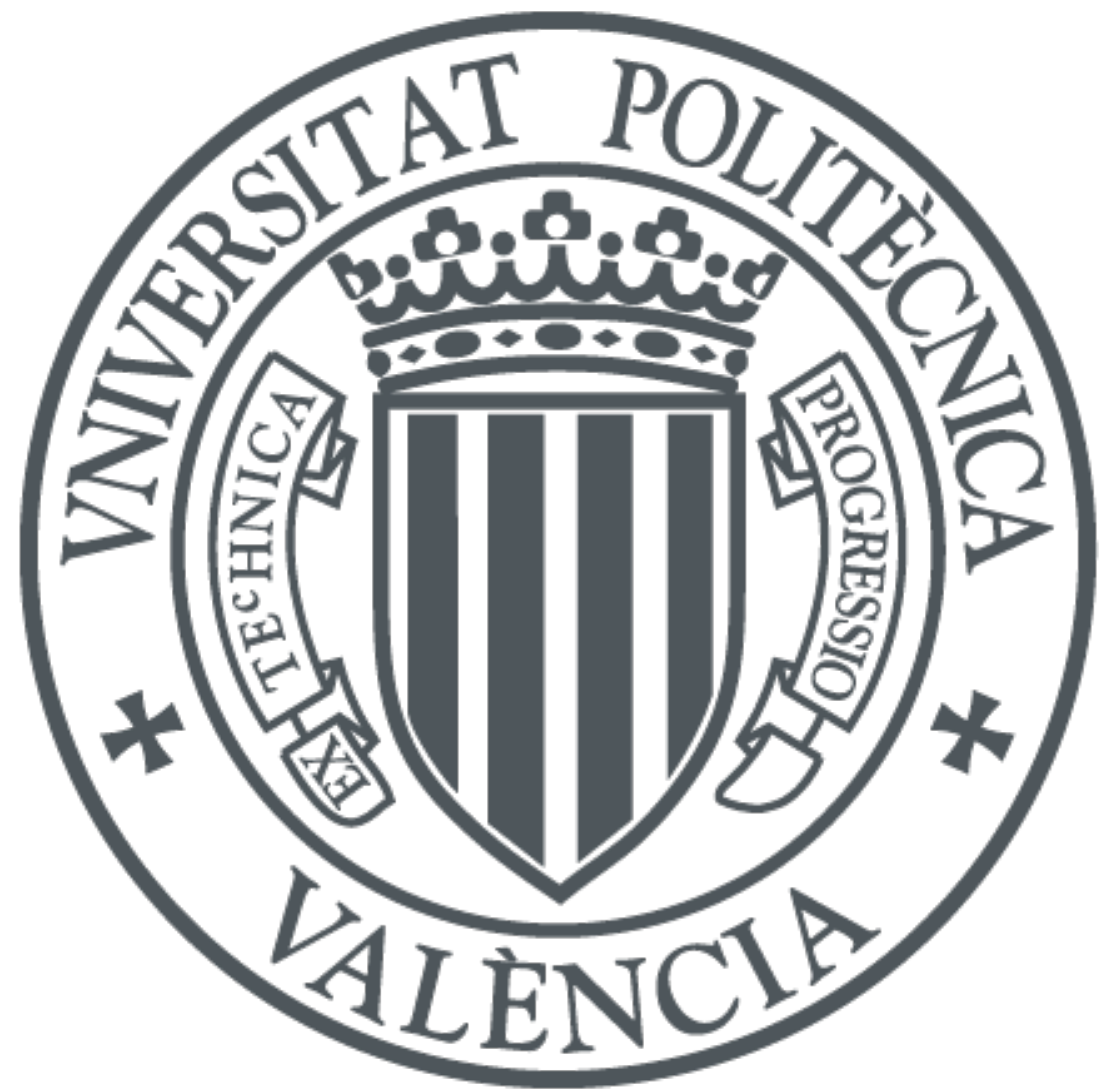

The final publication is available at

http://doi.org/10.1109/TMTT.2017.2769083

Copyright Institute of Electrical and Electronics Engineers

Additional Information 


\title{
Exploring the Tuning Range of Channel Filters for Satellite Applications using Electromagnetic-based Computer Aided Design Tools
}

\author{
Javier Ossorio, Joaquin Vague, Vicente E. Boria, and Marco Guglielmi
}

\begin{abstract}
The objective of this paper is to use Electromagnetic based Computer-Aided Design tools to investigate the maximum tuning range of channel filters, typically used in satellite payloads. Both circular and rectangular waveguide technologies are investigated. The results of the investigation show that single-mode rectangular waveguide implementations offer substantially wider tuning range, as opposed to classical dual-mode circular waveguide implementations. In addition to simulations, measurements are also presented indicating very good agreement with theory, thereby fully validating the Computer-Aided Design procedure.
\end{abstract}

Index Terms-Circular waveguide, dual-mode filter, electromagnetic-based computer aided design (EM-based CAD), rectangular waveguide, microwave filter, tunable, tuning.

\section{INTRODUCTION}

$\mathbf{T}$ HE basic structure of a 4-pole dual-mode filter in classical circular waveguide technology is shown in Fig. 1. This filter family was first introduced in [1], and has since become the structure of choice for the implementation of output multiplexers (OMUXes) for telecommunication satellites [2].

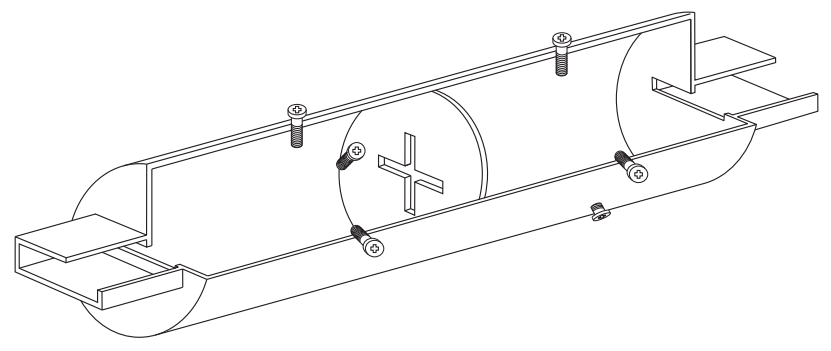

Fig. 1. Basic four-pole, dual-mode filter structure.

As a result, a very substantial amount of work can be found in the technical literature describing Computer-Aided Design (CAD) techniques for the design of circular waveguide dualmode filters (see, for instance [3]-[24]).

Manuscript received September 20, 2017; revised XXXXX XX, 2017; accepted XXXXX XX, 2017. Date of publication XXXXX XX 2017; date of current version XXXXX XX, 2017. This paper is an expanded version from the IEEE MTT-S International Conference on Numerical Electromagnetic Modeling and Optimization for RF, Microwave, and Terahertz Applications, May 17-19, 2017, Sevilla, Spain.

This work has been supported by MINECO (Spanish Goverment) under R\&D project TEC2016-75934-C4-1-R and R\&D Technicians grant PTA201510839-I.

All the authors are with Departamento de Comunicaciones, iTEAM, Universitat Politècnica de València, E-46022, Spain (e-mails: jaosgar@teleco.upv.es, jvague@dcom.upv.es, vboria@dcom.upv.es, marco.guglielmi@iteam.upv.es).
In addition, it is interesting to note that a number of contributions have also been published concerning dual, or singlemode rectangular waveguide filters, with filtering function similar to the ones of classic dual-mode filters in circular waveguide (see, for instance [25]-[35]).

This type of circular waveguide filter is commonly based on the $T E_{11 n}$ (with $n=1,3 \ldots$ ) dual resonant modes (with vertical and horizontal polarizations), where $n$ is chosen according to the filter requirements in terms of insertion loses and compactness [2]. They are usually designed to operate at a given center frequency and with a very narrow bandwidth (of $1 \%$ or less in relative terms). Usually, both center frequency and bandwidth are fixed to fit specific requirements.

Recently, however, the evolution of telecommunication satellite payloads is requiring the development of new filter structures that could allow for the remote variation of both filter center frequency and bandwidth [36]. In response to this new requirement, initial investigations have appeared in the technical literature discussing new, tunable dual-mode filter structures (see for instance [20], [37], and [38]). Furthermore, a very recent contribution [39], described the initial results of an investigation aimed at exploring the maximum tuning range for the class of filters based on the structure shown in Fig. 1.

In this context, therefore, the objective of this paper is to significantly extend the scope of the results presented in [39]. In particular, the proposed extension consists of:

1) The detailed CAD procedure for the structure shown in Fig. 1.

2) The detailed CAD procedure for an alternative singlemode rectangular waveguide structure that can be used to implement the same quasi-elliptic transfer function.

3) The details of the theoretical tuning range evaluation (in both center frequency and bandwidth) based on the Aggressive Space Mapping (ASM) approach [40] for both the circular and the rectangular waveguide filter structures.

4) A critical comparison of the performance of the two structures, including out of band results.

Our investigation will show that the rectangular waveguide implementation can offer a far superior tuning performance. Finally, in addition to Electromagnetic (EM) simulations, the measured performance of two four-pole filter breadboards are also discussed thereby fully validating the complete CAD procedure. 


\section{Circular Waveguide Dual-Mode Filters}

Fig. 2 shows the simulated behavior of the structure in Fig. 1. The performance shown in Fig. 2 has been obtained using the CAD tool FEST3D ${ }^{1}$, and filter design procedure described in [19]. The structure contains 6 tuning screws (see Fig. 1). However, for the sake of analysis, they have been represented by two lengths of uniform circular waveguide with three metallic ridges of rectangular cross section. This representation is indeed very convenient for the EM-Based CAD of the structure. This is because the sections containing the tuning screws can be easily analyzed as sections of uniform circular waveguide with three metal ridges (considered as perfect parallelepipeds).

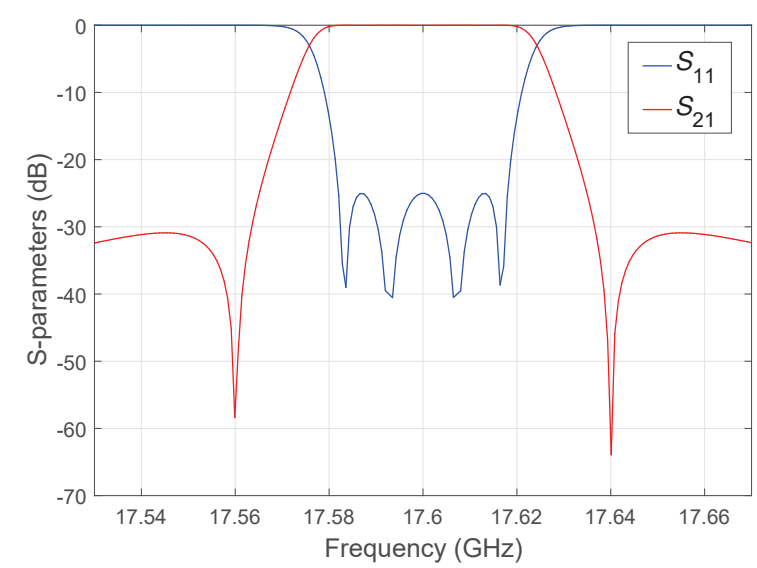

Fig. 2. Basic four-pole, dual-mode filter performance simulated with FEST3D.

It is now convenient to describe in more detail the (well known) physical behavior of the structure. In this context, we recall that the vertical and horizontal screws in Fig. 1 are used to tune the resonant frequency of the cavities, whereas the oblique screws are adjusted to implement the needed interresonator couplings. In principle, therefore, to tune the filter at another frequency, one could simply change the penetration of the six screws. This, however, is not sufficient by itself because, to change the operating frequency of the filter, we need to change also the dimensions of all coupling irises. To achieve full tuning, therefore, also the input-output and interresonator irises (see Fig. 3) must be tunable.

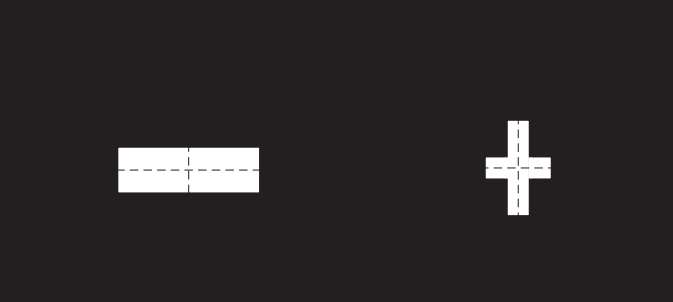

Fig. 3. Basic input/output and inter resonator irises.

As a matter of fact, the correct dimension of all coupling irises in standard (fixed frequency) dual-mode filter implementations is indeed a very critical problem, that normally requires extreme mechanical implementation accuracy.

\footnotetext{
${ }^{1}$ http://www.fest3d.com/
}

In the industrial practice, therefore, when tuning narrow band dual-mode filters, it may actually be necessary to try several irises before one can finally obtain the correct coupling values.

\section{A. Tunable Irises}

A first attempt at tuning an input-output iris, as the one shown in Fig. 3, can be found in [41]. The solution proposed in [41] was to insert a tuning screw in the center of the iris along the vertical direction of Fig. 3. This solution is indeed effective, but the achievable tuning range is very limited. In addition, placing a tuning screw vertically, may create serious multipaction problems in vacuum. A much better solution, allowing for a much wider tuning range, is the one proposed by Lamming [42], where the tuning screw is inserted laterally, along the horizontal direction, as shown in Fig. 4. In [42], however, the configuration in Fig. 4 was proposed in the context of a successful experimental study aimed only at recovering the errors introduced by the manufacturing process of the irises. In this work, we propose to extend the use of this iris tuning technique to the implementation of fully tunable dual-mode filters.

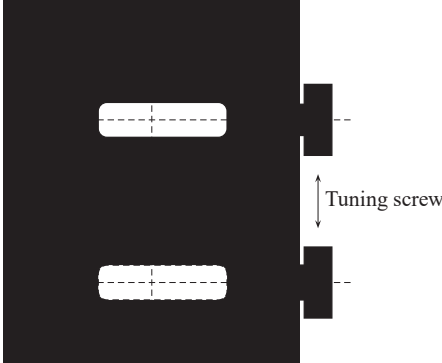

(a)

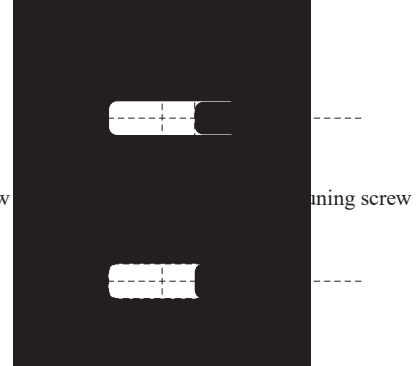

(b)
Fig. 4. Tunable input-output iris: (a) Non-penetrated screw; (b) Penetrated screw

The same tuning concept, however, can not be applied directly to the inter-resonator iris in Fig. 3. Inserting the tuning screws directly on the sides of the cross-shaped iris would result in much too long screws. To make the implementation of this simple concept more practical, it is better to split the cross iris into two separate offset irises, as shown in Fig. 5.

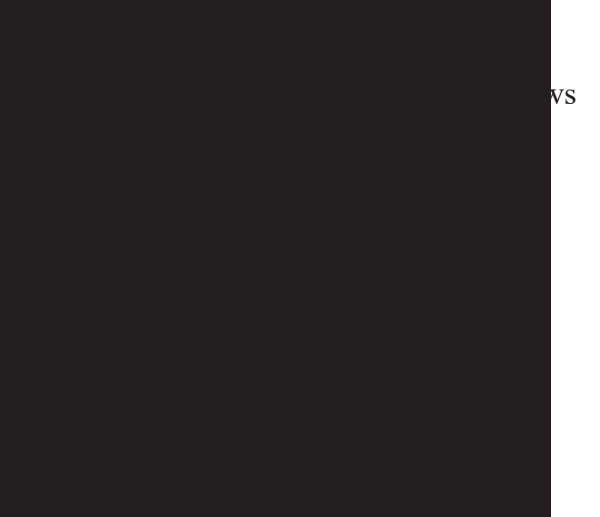

Fig. 5. Tunable inter resonator iris. 


\section{B. EM-Based CAD of the Tuning Screws}

As previously discussed, the full-wave EM simulations in support of this investigation have been carried using FEST3D. In this CAD tool, all waveguide structures are simply represented by cascaded sections of uniform waveguide, with the appropriate cross section. The junctions between the various sections of uniform waveguide are then represented by Multimode Equivalent Networks (MENs). Cascading the various MENs, we can then obtain the efficient and accurate full-wave EM-Based CAD model of the complete filter structure [43]. A similar approach can also be used to simulate the tunable irises in Figs. 4 and 5. In this case what we propose to do is simply to introduce a metallic cylinder of circular cross section (the real tuning screw) of the required length inside the waveguide length that represent the irises. The length of waveguide with the tuning screw inside can then be considered as a $3 \mathrm{D}$ rectangular cavity loaded with a post of circular cross section. The theory described in [43], to analyze arbitrary shaped 3D rectangular cavities, can then be used to produce an accurate MEN for the complete tunable iris. Fig. 6 shows the geometrical details of the resulting tunable input iris, where it can be observed that the diameter of the tuning screw is less than the iris thickness.

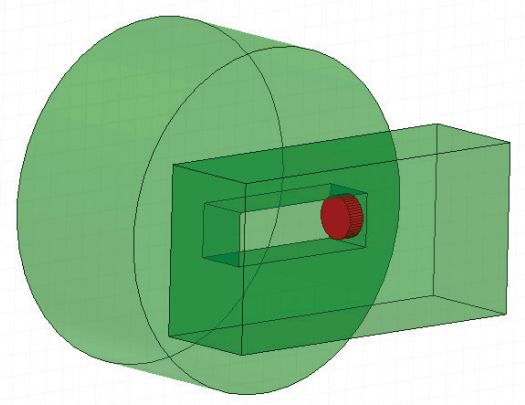

Fig. 6. Tunable input iris implementation.

Increasing the penetration of the metallic cylinder will reduce the width of the coupling iris, thereby reducing the coupling value. Reducing the penetration of the cylinder will, on the other hand, increase the width of the iris and increase the coupling value. There are, however, two important details that need further discussion. The first is that the diameter of the tuning cylinder must be less than the height of the window. This is because having the tuning cylinder and the window with the same height can introduce an electrical contact between the sides of the tuning cylinder and the walls of the irises. This electrical contact, however, cannot be easily kept stable. This instability would then introduce an unwanted filter performance. It must also be mentioned that the small air gaps between the screws and iris walls are not prone to induce multipaction, due to their size, physical location (low electric field intensity), and the effect of fringing fields [44]. The second is that the length of the tuning cylinder must, in any case, be kept smaller than the resonant length. This is because the resonance of the tuning cylinder would affect very negatively the response of the filter.

\section{Fully Tunable Dual-Mode Filter}

Implementing the tuning concepts described in the previous sections, we obtain the fully tunable filter structure shown in Fig. 7.

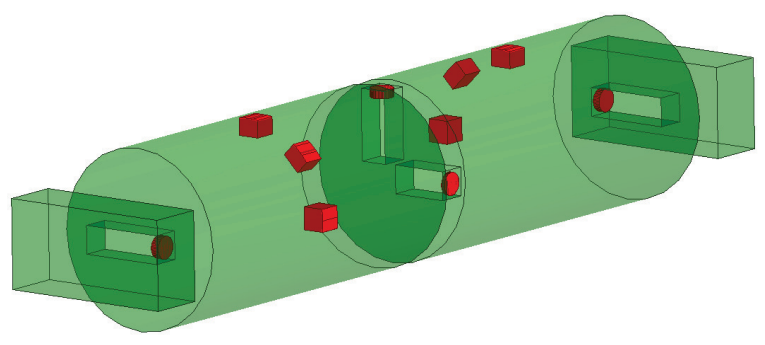

Fig. 7. Fully tunable dual-mode filter.

We can now use the CAD tool FEST3D to explore the maximum tuning range offered by the structure in Fig. 7 , keeping the bandwidth constant at $36 \mathrm{MHz}$. Fig. 8 shows the results obtained. The highest center frequency obtained is $17.64 \mathrm{GHz}$, whereas the lowest center frequency is 17.55 $\mathrm{GHz}$. A correctly tuned filter response can not be obtained above or below this frequency range.

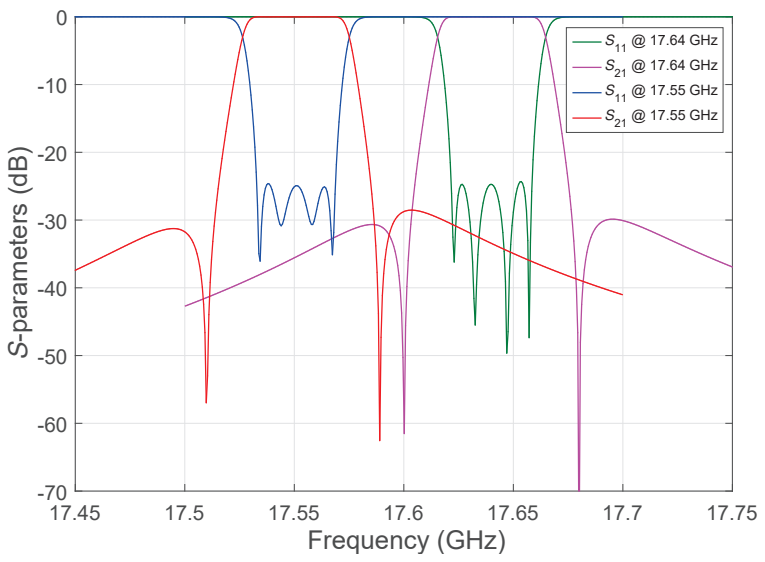

Fig. 8. Simulated tuning range with FEST3D.

To better understand this result, it is now convenient to look at the values of the penetration of the various screws, as shown in Table I. Note that all dimensions in Table I are in $\mathrm{mm}$. Furthermore, all apertures are $2.5 \mathrm{~mm}$ high and $2.5 \mathrm{~mm}$ thick. All tuning elements have $2 \mathrm{~mm}$ diameter. V stands for vertical, $\mathrm{H}$ stands for horizontal, $\mathrm{O}$ stands for oblique, VIR stands for vertical inter resonator, and HIR stands for horizontal inter resonator. Finally, when two values are given, the first is for the lower frequency, and the second is for the higher frequency.

Tuning the filter at lower frequencies, requires increasing the penetration of the tuning screws in the center of the cylindrical resonators. Tuning the filter below $17.55 \mathrm{GHz}$, however, brings the resonant frequency of the tuning screws themselves too close to the center frequency of the filter, so that the structure can not be effectively tuned. The horizontal tuning screw, in particular, needs to penetrate more and, therefore, becomes the limiting factor. 
TABLE I

FEST3D FILTER DIMENSIONS (MM)

\begin{tabular}{|c|c|c|c|}
\hline Filter element & Cavity 1 & Cavity 2 & Inter Cavity \\
\hline \hline I/O aperture & 7.2 & 7.2 & - \\
\hline I/O screw & $0.642-0.732$ & $0.635-0.772$ & - \\
\hline V screw & $1.537-1.012$ & $1.531-1.040$ & - \\
\hline H screw & $1.980-1.775$ & $1.983-1.770$ & - \\
\hline O screw & $1.472-0.974$ & $1.443-0.957$ & - \\
\hline VIR aperture & - & - & 5.9 \\
\hline VIR screw & - & - & $0.772-1.203$ \\
\hline HIR aperture & - & - & 4.7 \\
\hline HIR screw & - & - & $0.939-2.131$ \\
\hline
\end{tabular}

To better understand this point, we show in Fig. 9 the results of a simulation where we have a section of circular waveguide with only the horizontal tuning screw. The simulations have been performed for two values of tuning screw penetration, namely, $2.0 \mathrm{~mm}$ and $1.980 \mathrm{~mm}$ (this last one given in Table I for the $\mathrm{H}$ screw at the lower frequency). As we can clearly see, from Fig. 9, with $2 \mathrm{~mm}$ penetration, the resonance of the screw occurs within the tuning range of the dual-mode filter. As a consequence, the filter can no longer be tuned.

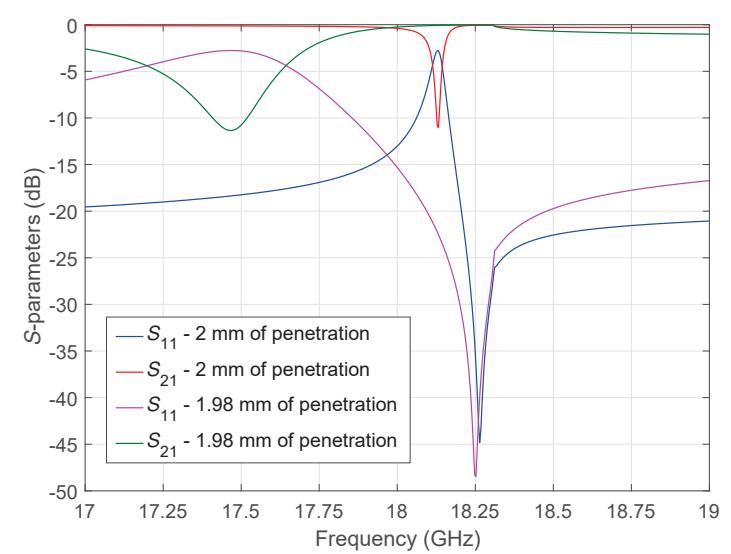

Fig. 9. Resonance of the horizontal screw.

On the other hand, tuning the filter at higher frequency would require decreasing the size of all coupling irises. However, above $17.64 \mathrm{GHz}$ the size of the HIR iris becomes too small and the filter cannot be tuned successfully. Above this frequency, the width of the VIR iris is large enough to provide also a significant negative coupling between the vertical modes, so that the HIR iris looses its effectiveness. This can be clearly appreciated by taking the difference between the HIR aperture and the HIR screw penetration for the higher frequency tuning value. The value obtained is 2.569 $\mathrm{mm}$, however, the width of the VIR iris is $2.5 \mathrm{~mm}$. It is therefore clear that if the HIR screw penetrates more, its effect will be marginal with respect to the width of the VIR iris. As a result, the center frequency cannot be further increased. In conclusion, the achieved tuning range turns out to be about $90 \mathrm{MHz}$. It is interesting to observe that the central frequency of the achieved tuning range is $17.595 \mathrm{GHz}$. Expressing the tuning range in terms of the center frequency $f_{0}$ would result into approximately $\Delta f_{0}= \pm 0.25 \%$ tuning range.
This is indeed the measure of the tuning range that is most commonly used in the technical literature. However, we propose an alternative definition of the tuning range, namely, in terms of the bandwidth of the filter:

$$
\Delta B W=\frac{f_{0, \max }-f_{0, \min }}{B W} \times 100
$$

Using this alternative definition results into approximately $\Delta B W_{c w}= \pm 125 \%$ tuning range for the circular waveguide filter (subscript $c w$ ). We do believe that this second definition is more meaningful than the first one, since it gives clear information about the movement in frequency of the filter with respect to its own nominal bandwidth.

The same conclusions -in terms of tuning range- have been observed for dual-mode circular waveguide filters providing standard Chebyshev responses (without the two adjacent transmission zeros), and for filter structures where symmetric arrangements are considered for the inter-resonator coupling iris (where two vertical and two horizontal slots are considered).

\section{Folded RECTANGULAR WAVEGUide IMPLEMENTATION}

As an alternative to the classic circular waveguide structure in Fig. 1, we propose to investigate in addition the folded rectangular waveguide implementation shown in Fig. 10.

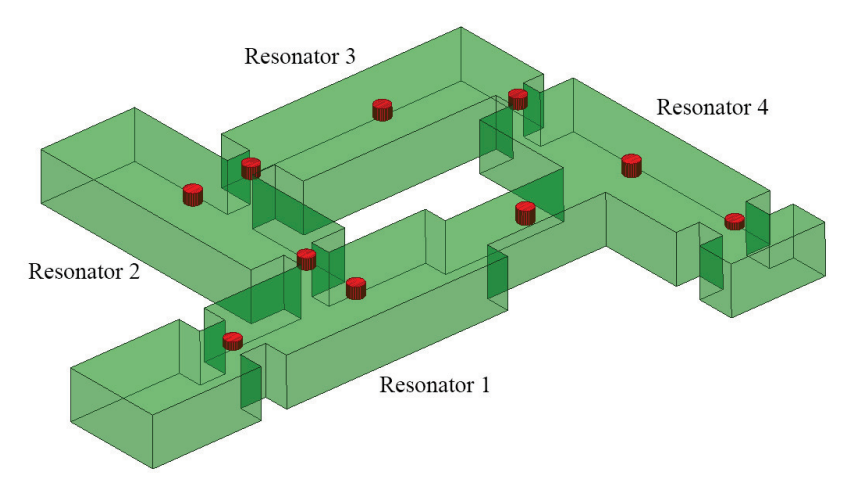

Fig. 10. Folded Rectangular waveguide filter.

The filter structure in Fig. 10 is based on the use of four $T E_{103}$ resonators, and can be designed to have an electrical performance that is approximately identical to the one shown in Fig. 2.

In particular, it is well known that in order to generate a pair of transmission zeros, it is necessary to have two signal paths with couplings of opposite signs. This can be easily achieved with the structure shown in Fig. 10, where the coupling between resonators 3 and 4 is positioned between the end of the third resonator and the beginning of the fourth one. Because of the alternating sign of the longitudinal field distribution of a $T E_{103}$ resonator, this arrangement results into a 3-4 coupling of opposite sign to the other ones (i.e. between resonators 1 and 2, resonators 2 and 3 , as well as between resonators 1 and 4 , where the coupling is always located between the middle of one resonator and the end of the other one). 
It is important to note that for the folded rectangular waveguide structure in Fig. 10, the tuning of the electrical parameters of interest, namely, the resonance frequencies and the coupling values, can very simply be achieved using standard vertical tuning screws in the center of the resonators, and in the center of the irises. The only limiting factor is the resonance of the tuning elements themselves. In this case, there are no significant interactions between different coupling apertures. The basic concept behind the tunable filter in Fig. 10 is simple. However, the tuning range of this type of structure must be investigated. Furthermore, it is interesting to note that a different tunable filter structure with similar performance has been discussed in the technical literature [46]-[48]. However, the structure in Fig. 10 is significantly simpler to manufacture and tune than the one proposed in [46]-[48].

\section{A. Center Frequency Tuning Range}

The first step is to design with FEST3D the filter at 17.6 $\mathrm{GHz}$, with $36 \mathrm{MHz}$ bandwidth, and two transmission zeros at $\pm 40 \mathrm{MHz}$ from the filter center frequency. The filter design procedure that we have followed in this case is based on the results presented in [45].

To explore the tuning range, we have then decreased the center frequency of the filter by $10 \mathrm{MHz}$ steps, and readjusted at each step all tuning screws. In Fig. 11 we show the simulated tuning range obtained with the folded rectangular waveguide structure in Fig. 10. As we can see from Fig. 11, the center frequency can be tuned from $17.6 \mathrm{GHz}$ down to 17.0 GHz. The achieved tuning range is therefore $600 \mathrm{MHz}$. Expressing this range in terms of equation (1) results into a tuning range equal to approximately $\Delta B W_{r w}= \pm 833 \%$.

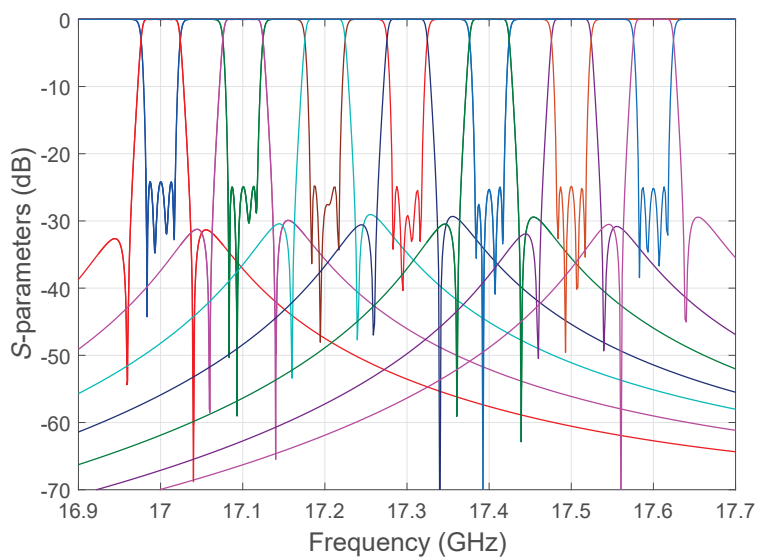

Fig. 11. Simulated tuning range of the folded rectangular waveguide filter.

This result is clearly far superior to the one obtained with the circular dual-mode filter $\left(\Delta B W_{c w}= \pm 125 \%\right)$. The limiting factor in this case is the lower center frequency, which is obtained when all tuning elements are fully inserted in the filter structure. To obtain a lower frequency, we would need to insert the tuning screws further into the structure. This, in turn, brings the resonance of the tuning element itself too close to the filter center frequency, and the desired filter performance can no longer be obtained.

\section{HARDWARE MANUFACTURE}

To verify the results of our full-wave simulations, we have built two filter prototypes, one in circular waveguide and the other in rectangular waveguide. Before building the filters, however, the basic designs in Figs. 7 and 10 have been slightly modified. The original (ideal) structures contain elements with sharp, 90 degrees corners. Although it is indeed possible to build hardware with sharp corners, in the industrial practice it is preferred to use rounded corners. This has two main effects, namely, it decreases the manufacturing cost of the hardware, and improves the quality of the silver plating that is generally used to achieve low insertion losses. Furthermore, the rectangular cross section tuning elements in the center of the cavities of the dual-mode filter have been replaced with circular cross section tuning screws.

The transition between the ideal sharp corner structures and the real hardware with rounded corners has been performed using the ASM procedure [40], using FEST3D (with the ideal structures) as the low accuracy (LA) space, and ANSYS HFSS and the real hardware as the high accuracy (HA) space. The resulting filter geometries are shown in Fig. 12 and 13 for the circular and rectangular waveguide filters, respectively. In this context, it is important to note that FEST3D has the very useful feature of allowing the user to set the accuracy of the simulations by changing the values of a set of computational parameters.

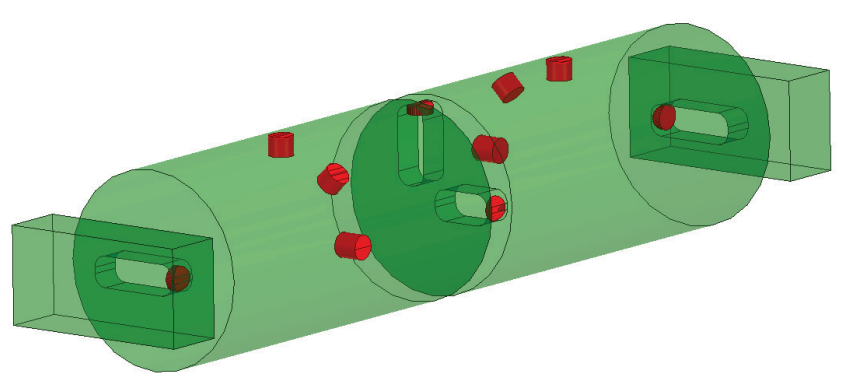

Fig. 12. Fully tunable circular waveguide filter with rounded corners irises and all real tuning screws.

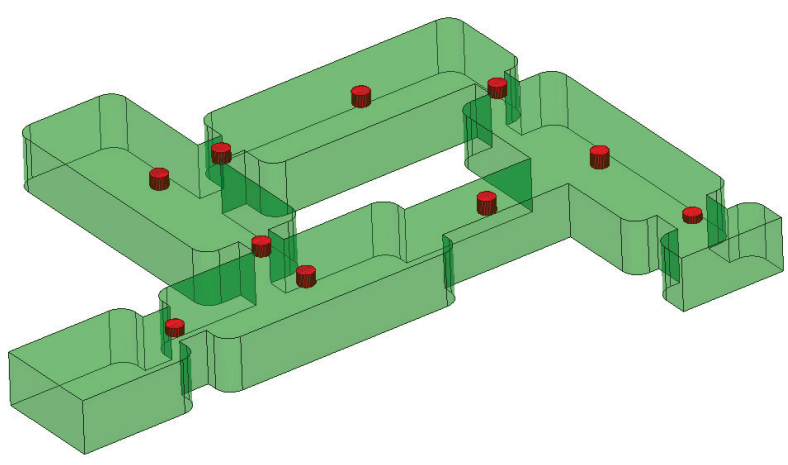

Fig. 13. Folded tunable rectangular waveguide filter with rounded corners and real tuning screws. 
Naturally, in the low accuracy mode, the computations are much faster than in the high accuracy mode. In fact, all the simulations discussed in this paper so far have been carried out using FEST3D in the low accuracy mode. This has allowed us to carry out in a very short time the very many optimizations required to achieve the (ideal) performances that we have shown in the previous sections. Figs. 14 and 15 show the comparison between the simulated response of the ideal LA models and the (real) HA performances obtained at one single center frequency.

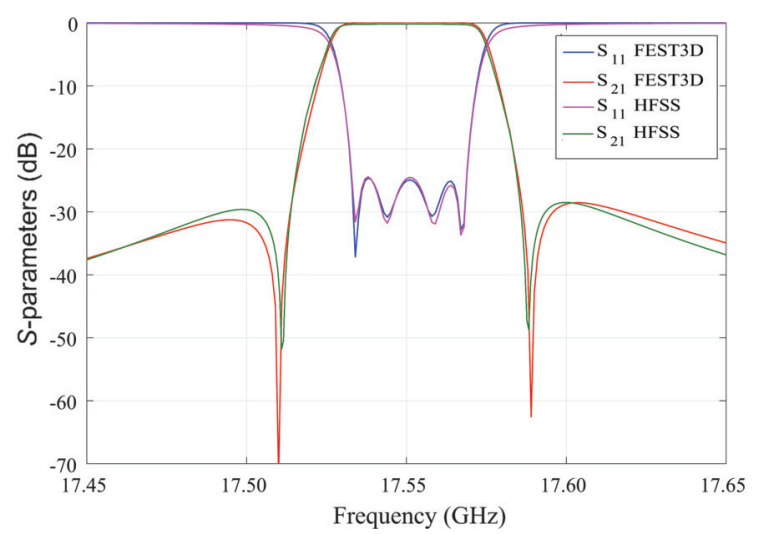

Fig. 14. Comparison between simulated response in FEST3D and HFSS of the dual-mode circular waveguide filter, tuned at the center frequency.

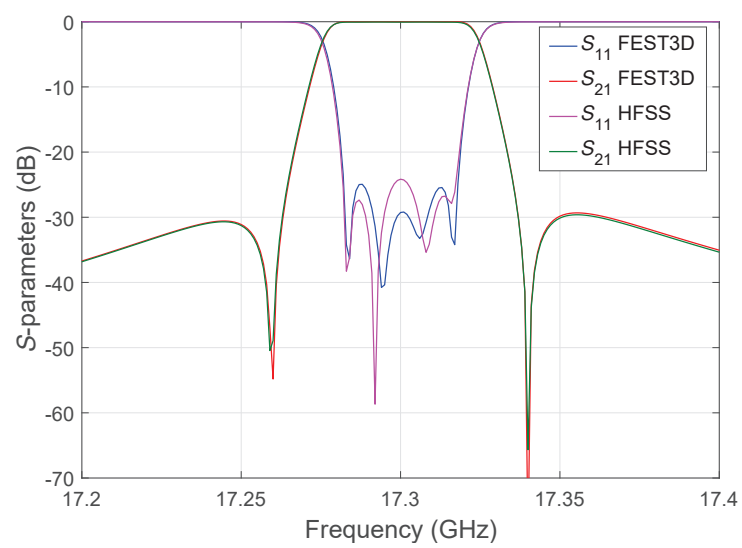

Fig. 15. Comparison between simulated response in FEST3D and HFSS of the folded rectangular filter, tuned at the center frequency

As we can see, the two results are almost exactly coincident so that the LA and the HA spaces can be considered to be perfectly aligned. Fig. 16 and 17 show the real hardware manufactured.

As we will show in the next section, once the LA and the HA spaces are aligned, the real hardware will behave as indicated by the simulations in the LA space. In practice, the changes in filter performance will be obtained by changing the penetration of the tuning elements by a very small amount. We therefore expect the real hardware to behave as indicated by the simulations in the LA space as a consequence of small dimensional changes. This is, in fact, the very same concept that is at the core of the ASM procedure.

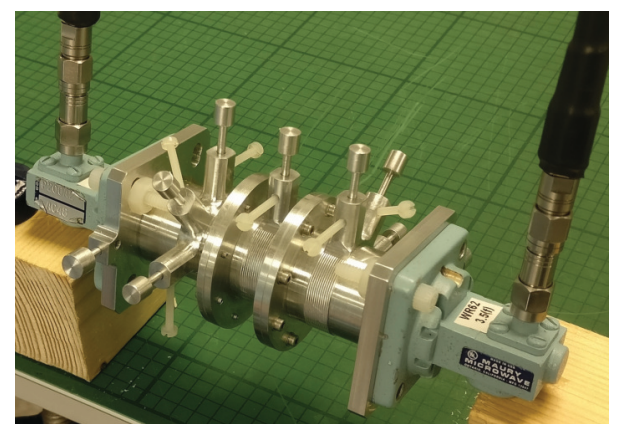

Fig. 16. The tunable four-pole circular waveguide filter breadboard.

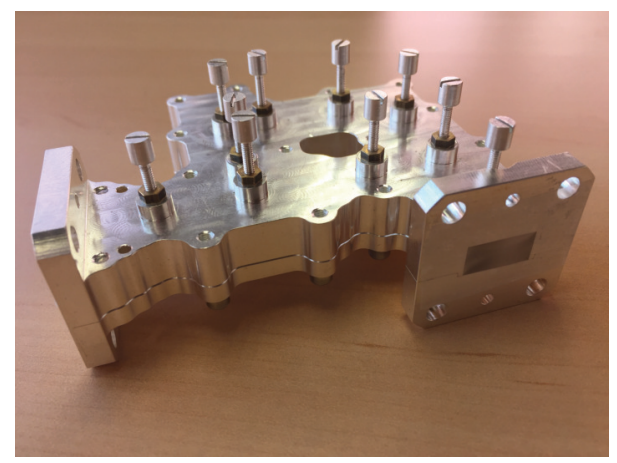

Fig. 17. Folded rectangular waveguide filter realized in silver plated aluminum.

\section{Measured Results}

Once the hardware has been manufactured, we have carried out two sets of measurements. The first is to evaluate the center frequency tuning range, and the second to evaluate the bandwidth tuning range, of both prototypes. The results obtained are reported in the next sections.

\section{A. Center Frequency Tunability}

Fig. 18 shows the measured results obtained with the dualmode filter made of silver plated aluminum. In all measured responses, the in-band return losses are around $25 \mathrm{~dB}$.

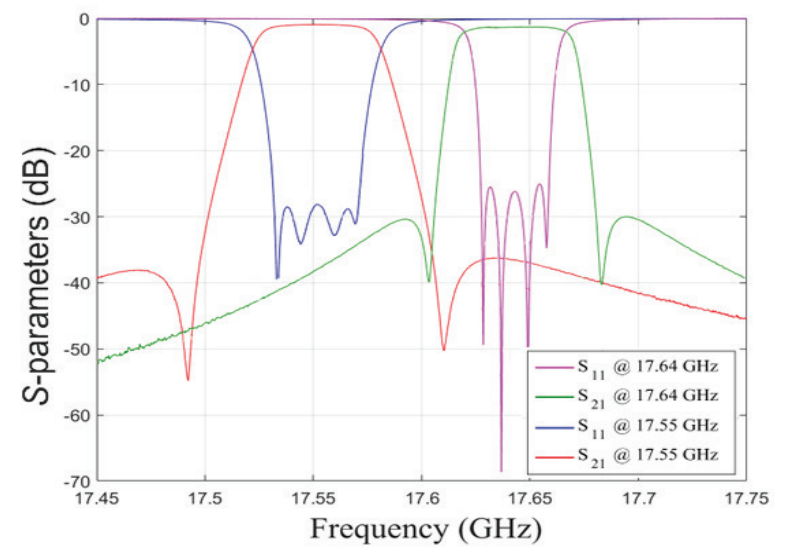

Fig. 18. Measured tuning range of the circular waveguide dual-mode filter. 
As we can see, although the higher frequency filter is slightly narrower in bandwidth (due to manufacturing tolerances observed in the practical implementation of filter irises), the basic tuning performance achieved is the same one as indicated by the LA simulations in Fig. 8.

The measured performance of the folded rectangular waveguide filter is shown in Fig. 19.

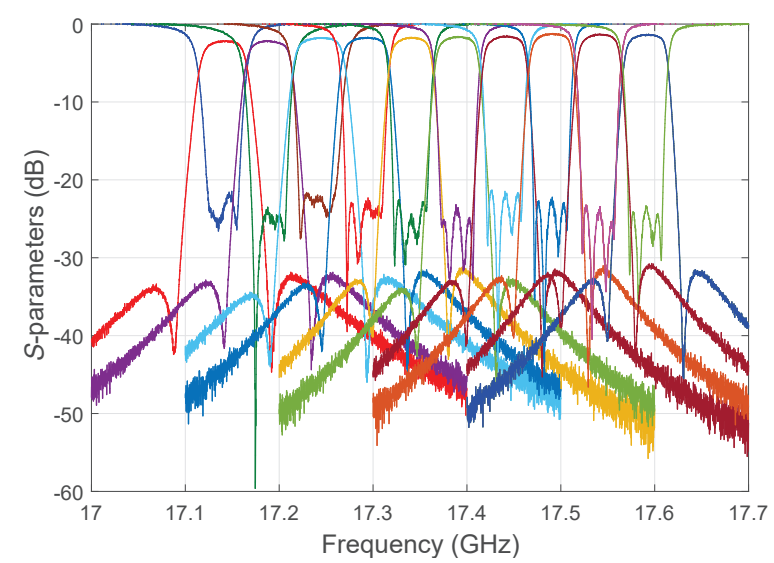

Fig. 19. Measured tuning range of the folded rectangular waveguide filter.

As we can see, the tuning range achieved (keeping always return losses above $22 \mathrm{~dB}$ ) is about $550 \mathrm{MHz}$, resulting in a measured tuning range of about $\Delta B W_{r w}= \pm 763 \%$. The small difference between the simulated $(600 \mathrm{MHz})$ and the measured range is probably due to the manufacturing tolerances.

\section{B. Bandwidth Tuning Range}

Similarly, to what we have done for the center frequency tuning, we have also explored the maximum tuning range for the bandwidth of the two filters (that has been recovered at the central frequency of each tuning range).

The maximum measured bandwidth for the silver plated dual mode filter turned out to be about $51 \mathrm{MHz}$, resulting in a tuning range greater than $42 \%$ (Fig. 20).

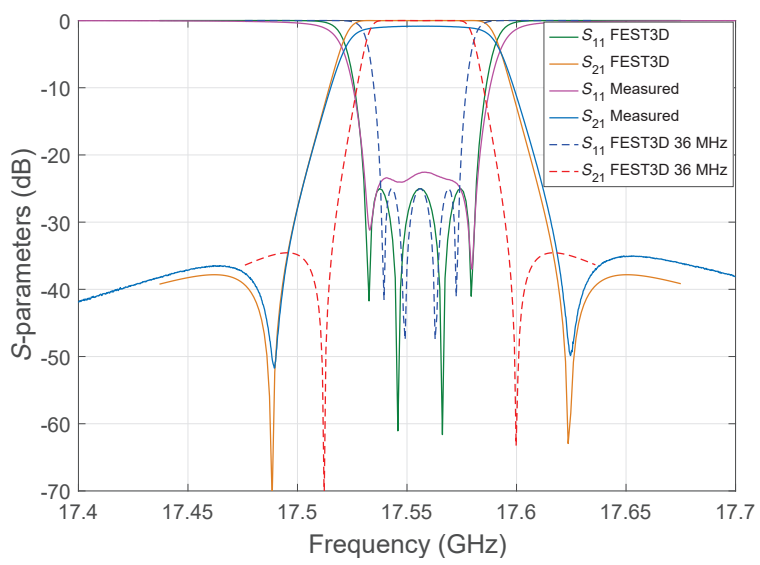

Fig. 20. Maximum bandwidth for circular waveguide dual mode filter.
Finally, we show in Fig. 21 the bandwidth tuning of the folded rectangular waveguide filter. As we can see, the achieved bandwidth is now $69 \mathrm{MHz}$, resulting in a tuning range greater than $92 \%$.

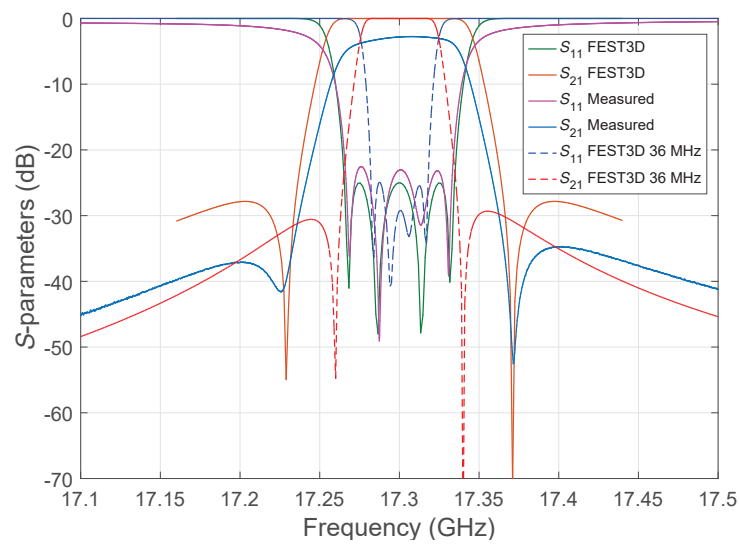

Fig. 21. Maximum bandwidth for folded rectangular waveguide dual mode filter.

\section{COMPARATIVE Discussion}

The results obtained so far can now be summarized in a comparative discussion. Three aspects will be addressed, namely tuning, insertion losses, and out of band performance.

\section{A. Tuning Range}

As we can clearly see by comparing the measured results in Figs. 18 and 19, the single-mode rectangular waveguide implementation can achieve a far superior tuning range than the dual-mode circular waveguide implementation. The reason for this difference is the fact that the use of dual modes imposes conditions on the tuning screw penetrations that result into a limited tuning range. Furthermore, the presence of two resonances in a single cavity introduces also interactions between coupling apertures that further limit the tuning range.

In the single-mode implementation, on the other hand, there are essentially no interactions between tuning screws or coupling apertures, so that the tuning screws can be inserted in the structure to the maximum allowed, and the tuning range results significantly extended. The only limiting factor in the single-mode case is the resonance of the tuning elements themselves.

\section{B. Insertion Losses}

As expected, the circular waveguide implementation achieves lower insertion loss $(0.5 \mathrm{~dB})$ than the rectangular waveguide implementation $(2.5 \mathrm{~dB})$.

This result is clearly due to the lower quality factor of rectangular waveguide resonators as opposed to the circular waveguide resonators. It is interesting to note, however, that the height of the rectangular waveguide filter can be increased and this, in turn, can lower the insertion losses, thus bringing it to values that are comparable to the ones achieved by circular waveguide [45]. 
Obviously, the tuning of this new filter prototype would be achieved with other penetration depths (slightly higher than the previous ones) of the corresponding screws.

\section{Out of Band Performance}

One aspect of the performance of this type of filter that is of key importance is the out of band response. This is particularly important if the filters are to be used in an output multiplexer. Fig. 22 shows the comparison of the out of band performance of the two filter types. It has been checked that the out of band behavior of the proposed circular waveguide dual-mode filter is very similar to the one for the same kind of filter using a classical cross-shaped iris (instead of the one used in this work, see Fig. 5), thus confirming that the asymmetric tunable inter resonator iris we use does not generate additional spurious resonant modes.

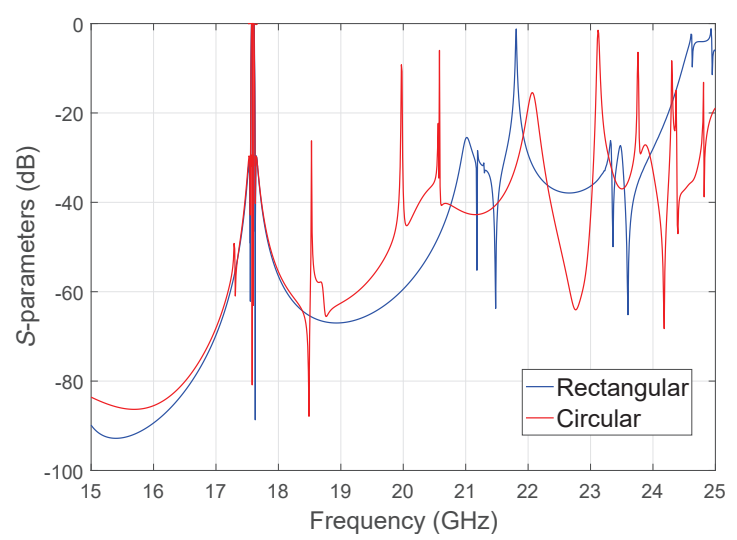

Fig. 22. Out of band performance comparison. The red curve is the circular waveguide dual-mode filter.

As we can see, the folded rectangular waveguide filter has a substantially cleaner out of band response.

Finally, it is important to note that an alternative configuration for implementing tunable channel filters could indeed be based on a folded configuration using single-mode circular waveguide resonators, coupled through rectangular irises. This structure could probably offer a competitive performance in terms of tuning range and insertion losses, but the limitation in terms of out-of-band performance would still be present.

\section{CONCLUSION}

EM-Based CAD techniques have been used in this paper to explore the maximum tuning range of two output channel filter structures. Namely, a classical dual-mode filter in circular waveguide, and a folded rectangular waveguide filter. The results of our investigation clearly show that the folded rectangular waveguide implementation has a measured center frequency tuning performance $\left(\Delta B W_{r w}= \pm 763 \%\right)$ that is far superior to the one of the dual-mode circular waveguide implementation $\left(\triangle B W_{c w}= \pm 125 \%\right)$. Furthermore, the bandwidth tuning of the rectangular waveguide filter is also superior $(92 \%)$ to the one of the circular waveguide dual-mode filter $(42 \%)$. Finally, measured results have also been presented showing very good agreement with simulations, thereby fully validating all the results presented.

\section{REFERENCES}

[1] A. E. Williams, "A four-cavity elliptic waveguide filter", IEEE Trans. Microw. Theory Techn., vol. MTT-18, no. 12, pp. 1109-1114, Dec. 1970.

[2] R. Cameron, C. Kudsia, and R. Mansour, Microwave Filters for Communication Systems: Fundamentals, Design and Applications. New York, NY, USA: Wiley, Aug. 2007.

[3] M. Guglielmi, R. C. Molina, and A. A. Melcon, "Dual-mode circular waveguide filters without tuning screws", IEEE Microwave and Guided Wave Letters, vol. 2, no. 11, pp. 457 - 458, Nov. 1992.

[4] R. Beyer and F. Arndt, "Field-theory design of circular waveguide dualmode filters by a combined mode-matching finite element method", Proc. 1994 24th European Microwave Conf., vol. 1, pp. 294 - 303, Sept. 1994.

[5] S. Moretti, F. Alessandri, and R. Sorrentino, "Field theory design of a novel circular waveguide dual-mode filter", Proc. 1995 25th European Microwave Conf., vol. 2, pp. 779 - 783, Sept. 1995.

[6] J. R. Montejo-Garai, and J. Zapata, "Full-wave design and realization of multicoupled dual-mode circular waveguide filters", IEEE Trans. Microw. Theory Techn., vol. 43, no. 6, pp. 1290 - 1297, Jun. 1995.

[7] L. Accatino, G. Bertin, and M. Mongiardo, "A four-pole dual-mode elliptic filter realized in circular cavity without screws", IEEE Trans. Microw. Theory Techn., vol. 44, no. 12, pp. 2680 - 2687, Dec. 1996.

[8] W. Schroeder and M. Guglielmi, "Boundary integral equation approach to multi-mode Y-matrix characterization of multi-ridged sections in circular waveguide", 1996 IEEE MTT-S International, Microwave Symp. Dig., vol. 3, pp. 1849 - 1852, Aug. 1996.

[9] P. Mezzanotte, F. Alimenti, L. Roselli, and S. Sordi, "Design and realization of a dual-mode circular waveguide filter for Digital Audio Broadcasting (DAB) applications", Proc. 1997 27th European Microwave Conf., vol. 2, pp. 773 - 77, Mar. 1997.

[10] V. E. Boria, M. Guglielmi, and P. Arcioni, "Accurate CAD for dualmode filters in circular waveguide including tuning elements", 1997 IEEE MTT-S International Microwave Symp. Dig., vol. 3, pp. 1575 - 1578, Aug. 1997.

[11] K. Wu, "An optimal circular waveguide dual-mode filter without tuning screws", IEEE Trans. Microw. Theory Techn., vol. 47, no. 3, pp. 271 276, Mar. 1999.

[12] K. Wu, M. Yu, and A. Sivadas, "Novel modal analysis of a circular-torectangular waveguide T-junction and its application to design of circular waveguide dual-mode filters", IEEE Trans. Microw. Theory Techn., vol. 50, no. 2, pp. 465 - 473, Aug. 2002.

[13] W. Steyn and P. Meyer, "A shorted waveguide-stub coupling mechanism for narrow-band multimode coupled resonator filters", IEEE Trans. Microw. Theory Techn., vol. 52, no. 6, pp. 1622 - 1625, Jun. 2004.

[14] M. Taroncher et. al, "CAD of complex passive devices composed of arbitrarily shaped waveguides using nyström and BI-RME methods", IEEE Trans. Microw. Theory Techn., vol. 53, no. 6, pp. 2153 - 2163, Jun. 2005.

[15] J. Zheng and M. Yu, "Rigorous mode-matching method of circular to off-center rectangular side-coupled waveguide junctions for filter applications", IEEE Trans. Microw. Theory Techn., vol. 55, no. 11, pp. 2365 - 2373, Nov. 2007.

[16] M. Bekheit, S. Amari, "A direct design technique for dual-mode in-line microwave bandpass filters", IEEE Trans. Microw. Theory Techn., vol. 57, no. 9, pp. 2193 - 2202, Aug. 2009.

[17] $\mathrm{H}$. Hu and K. Wu, "An automated design technique for asynchronouslytuned circular waveguide dual-mode filters", Proc. 2010 Asia-Pacific Microwave Conf., pp. 1970 - 1973, Mar. 2010.

[18] H. Hu, K. Wu, and R. J. Cameron, "A design technique for stepped circular waveguide dual-mode filters for broadband contiguous multiplexers", Proceedings 2011 IEEE MTT-S International Microwave Symposium, pp. 1 - 4, Aug. 2011.

[19] S. Cogollos et. all, "A systematic design procedure of classical dualmode circular waveguide filters using an equivalent distributed model", IEEE Trans. Microw. Theory Techn., vol. 60, no. 4, pp. 1006-1017, Apr 2012.

[20] W. Han, Q. Wang, Y. Chen, and Y. Tan, "Design of a narrow-band dual-mode circular waveguide filter", The 2012 International Workshop on Microwave and Millimeter Wave Circuits and System Technology (MMWCST), pp. 1 - 3, Apr. 2012.

[21] H. Hu, K. Wu, and R. J. Cameron, "Stepped circular waveguide dualmode filters for broadband contiguous multiplexers", IEEE Trans. Microw. Theory Techn., vol. 61, no. 1, pp. 139 - 145, Dec. 2013.

[22] $\mathrm{H}$. Hu and K. Wu, "A deterministic EM design technique for general waveguide dual-mode bandpass filters", IEEE Trans. Microw. Theory Techn., vol. 61, no. 2, pp. 800 - 807, Dec. 2013. 
[23] B. Yassini and M. Yu, "A novel Ka band dual-mode super Q cavity filter”, Proc. 2014 IEEE MTT-S International Microwave Symp. (IMS2014), pp. 1 - 3, Jul. 2014.

[24] C. Tomassoni, M. Dionigi, and R. Sorrentino, "Strategies for the improvement of the out of band behavior of TM dual-mode filters", 2015 IEEE 1st International Forum on Research and Technologies for Society and Industry Leveraging a better tomorrow (RTSI), pp. 90 - 93, Nov. 2015.

[25] X.g Liang, K. A. Zaki, and A. E. Atia, "Dual-mode coupling by square corner cut in resonators and filters", IEEE Trans. Microw. Theory Techn., vol. 40, no. 12, pp. 2294 - 2302, Dec. 1992.

[26] J. Liang, X. Liang, K. A. Zaki, and A. E. Atia, "Dual-mode dielectric or air-filled rectangular waveguide filters", IEEE Trans. Microw. Theory Techn., vol. 42, no. 7, pp. 1330 - 1336, Jul. 1994.

[27] M. Guglielmi, P. Jarry, E. Kerherve, O. Roquebrun, and D. Schmitt, "A new family of all-inductive dual-mode filters", IEEE Trans. Microw. Theory Techn., vol. 49, no. 10, pp. 1764 - 1769, Oct. 2001.

[28] J. A. Ruiz-Cruz, Y. Zhang, J. R. Montejo-Garai, J. M. Rebollar, and K. A. Zaki, "Longitudinal dual-mode filters in rectangular waveguide", 2008 IEEE MTT-S International Microwave Symp. Dig., pp 631 - 634, Sept. 2008.

[29] M. M. Darwish, A. M. El-Tager, H. N. Ahmed, and H. S. El-Hennawy, "Design of in-line dual-mode rectangular waveguide bandpass filters using multiple inductive circular posts", Proc. 2008 38th European Microwave Conf., pp. 500 - 503, Jan. 2008.

[30] R. Sorrentino, L. Pelliccia, and S. Bastioli, "Recent progress in miniaturized and reconfigurable filters for advanced communications and space applications", Proc. 2011 Microwaves, Radar and Remote Sensing Symp., pp. 20 - 23, Oct. 2011.

[31] Z. Zheng, J. Hu, S. Liu, and Y. Zhang, "WR-1.5 band waveguide bandpass dual-mode filter on silicon micromachining technique", 2015 IEEE International Conf. on Comm. Problem-Solving (ICCP), pp. 112 11, Apr. 2015

[32] S. Liu, J. Hu, Z. Xuan, Y. Zhang, and R. Xu, "Micromachined WR1.0 waveguide band-pass filter", 2016 IEEE International Conf. on Microwave and Millimeter Wave Technology (ICMMT), vol. 2, pp. 949 951, Dec. 2016.

[33] C. Kelleci and A. Atalar, "An analytical approach to the design of multiple mode rectangular cavity waveguide filters", IEEE Trans. Microw. Theory Techn., vol. 65, no. 8, pp. 2857 - 2865, Mar. 2017.

[34] Wang Junlin, Zhang Binzhen, Wang Xin, Duan Junping, and Wang Wanjun, "Dual-mode band-pass filters made by SU- 8 micromachining technology for terahertz region", Electron. Lett., vol. 53, no. 11, pp. 730 - 732, May, 2017.

[35] L. Accatino and G. Macchiarella, "A compact waveguide filtering structure with transmission zeros for multi-beam satellites", Proceedings 2017 IEEE MTT-S International Microwave Symposium (IMS2017)

[36] H. Leblond et. all, "When new needs for satellite payloads meet with new filters architecture and technologies", Proc. of the 39th European Microwave Conf., pp. 1712-1715, Oct. 2009

[37] U. Rosenberg et. all, "Reconfigurable doublet dual-mode cavity filter designs providing remote controlled center frequency and bandwidth reallocation", Proc. of the 46th European Microwave Conf., pp. 532-535, Jan. 2016.

[38] L. Accattino, G. Macchiarella, and G. Bertin, "Modelling techniques for dual-mode reconfigurable filters used in satellite applications", Proc. of the IEEE MTT-S International Conf. on Numerical Electromagnetic and Multiphysics Modeling and Optimization (NEMO2016), pp. 1-2, Jul. 2016.

[39] J. Ossorio, J. Vague, V.E. Boria, and M. Guglielmi, "Tunability range of dual-mode filters", Proc. of the IEEE MTT-S International Conference on Numerical Electromagnetic and Multiphysics Modeling and Optimization (NEMO2017), pp. 362-364, Jul. 2017.

[40] A. Rodriguez et. all, "Robust optimization and tuning of microwave filters and artificial transmission lines using aggressive space mapping techniques", Proc. 2017 IEEE MTT-S International Microwave Symp. (IMS2017), Oct. 2017.

[41] M.H.N. Potok, "Capacitive-iris-type mechanically tunable waveguide filters for the X-band", Proc. of the IEEE, Electronic and Communication Engineering, vol. 109, no. 48, pp. 505-510, Nov. 1962.

[42] M.A. Lamming, "Tunable irises for waveguide channel filters", Proc. of the ESA Workshop on Advanced CAD for Microwave Filters and Passive Devices, pp. 301-315., Noordwijk, NL, Nov. 1995.

[43] G. Conciauro, M. Guglielmi, and R. Sorrentino, ”Advanced Modal Analysis", New York, NY, USA: Wiley, Dec. 1999.
[44] P. Mader et. all, "Experimental validation of fringing field effects for the multipactor phenomenon", Proc. of 2012 International Symp. on Antenna Technology and Applied Electromagnetics, pp. 1-5, Aug. 2012.

[45] F. Alessandri, M. Comparini, M. Guglielmi, D. Schmitt, and F. Vitulli, "Low-loss filters in rectangular waveguides", Microwave and Optical Technology Lett., no. 1, Oct. 2000.

[46] C. Arnod, J. Parlebas, and T. Zwick, "Center frequency and bandwidth tunable waveguide bandpass filter with transmission zeros", Proc. of the 10th European Microwave Integrated Circuits Conference (EuMIC), pp. 369-372, Sept. 2015

[47] C. Arnold, J. Parlebas, and T. Zwick, "Reconfigurable waveguide filter with variable bandwidth and center frequency", IEEE Trans. Microw. Theory Techn., vol. 62, no. 8, pp. 1663-1670, Aug. 2014

[48] C. Arnold, J. Parlebas, Richard Meiser, and T. Zwick, "Fully Reconfigurable Manifold Multiplexer", IEEE Trans. Microw. Theory Techn., vol 65, no. 10 , pp. 3885 - 3891, Apr. 2017. 